\title{
Fiberoptic Endotracheal Intubation After Topicalization with In-Circuit Nebulized Lidocaine in a Child with a Difficult Airway
}

\author{
Ban C. H. Tsui, MD, MSc, FRCP(C), and Kirsten Cunningham, MB ChB \\ *Department of Anesthesiology and Pain Medicine, University of Alberta, Edmonton, Alberta, Canada
}

This case report describes the successful fiberoptic intubation of an uncooperative child with a difficult airway due to gross burn scarring in the facial and neck region by administering $4 \%$ end-tidal sevoflurane and simultaneously delivering $4 \%$ nebulized lidocaine via a small-volume nebulizer that was connected to the inspiratory limb of the circle system via a T-piece adapter. This case suggests that simultaneously administering a volatile anesthetic with nebulized lidocaine might be an alternative way to deliver lidocaine and might provide better topical anesthesia for uncooperative patients.

(Anesth Analg 2004;98:1286-8)
D ifficult airways in pediatric anesthesia may be managed by using inhaled induction followed by the topical application of local anesthesia to the airway in spontaneously breathing patients. This technique requires precise timing and an appropriate depth of anesthesia, because stimulation of the airway in a child who is only lightly anesthetized increases the potential for desaturation and hypoxemia due to coughing and laryngospasm. Waiting too long before manipulation of the airway could increase the partial pressure of the volatile anesthetic in the body and result in apnea and bradycardia. This report describes the successful fiberoptic intubation of an uncooperative child after the airway was anesthetized with $4 \%$ nebulized lidocaine and simultaneous administration of a volatile anesthetic.

\section{Case Report}

An 8-yr-old boy was scheduled for a repeated operation of scar releasing and skin grafting of the nasal, facial, and neck region (Fig. 1). Two years previously, the patient had sustained a burn involving $65 \%-70 \%$ of his total body-surface

Supported in part by the Education and Research Fund, Department of Anesthesiology and Pain Medicine, University of Alberta Hospitals, Edmonton, Alberta, Canada; and the Clinical Investigatorship Award, Alberta Heritage Foundation for Medical Research, Alberta, Canada.

Accepted for publication November 4, 2003.

Address correspondence and reprint requests to Ban C. H. Tsui, MD, MSc, FRCP(C), Department of Anesthesiology and Pain Medicine, University of Alberta Hospitals, 3B2.32 Walter Mackenzie Health Science Centre, 8440-112 St., Edmonton, Alberta, Canada T6G 2B7. Address e-mail to btsui@ualberta.ca.

DOI: 10.1213/01.ANE.0000108486.70256.E8 area during a gas explosion. In addition, this patient had chromosome $9 \mathrm{p}$ duplication syndrome, which is associated with global developmental delay, mental retardation, and characteristic craniofacial and skeletal malformations. The patient also had a history of difficult airway management. A laryngeal mask (LMA) was required for a previous operation immediately after inhaled induction because the patient became apneic and did not maintain a patent airway with positive mask ventilation. After multiple failed intubation attempts by direct laryngoscopy, fiberoptic intubation via LMA was successful.

The physical examination revealed a frightened and badly scarred boy weighing $20 \mathrm{~kg}$, with severe contractures in the face and neck region. There was an obvious limitation of mouth opening and a deformity of the neck, which was characteristically short with virtually no flexion or extension. His nostrils were also not patent.

The anesthetic plan was to attempt fiberoptic intubation after inhaled induction with sevoflurane. In the event that the airway became lost, our plan was to secure the airway with an LMA, which had been successful in the past. Tracheostomy was also considered if there was any compromise of patient safety. Equipment for this was kept ready in the operating room.

Anesthetic induction was performed with $8 \%$ sevoflurane with $100 \%$ oxygen. Transient apnea occurred but quickly resolved by reduction of sevoflurane to $4 \%$ end-tidal. The patient was maintained with $4 \%$ end-tidal sevoflurane. A 22-gauge IV line was established, and the patient received IV glycopyrrolate $0.01 \mathrm{mg} / \mathrm{kg}$. After $5 \mathrm{~min}$, an adequate depth of anesthesia was reached, and the nebulization of $4 \%$ lidocaine with auxiliary oxygen at a flow rate of $4 \mathrm{~L} / \mathrm{min}$ was initiated (Fig. 2). The nebulization was discontinued after exactly $5 \mathrm{~min}$, and fiberoptic intubation was then performed. The child tolerated the procedure well, without coughing or desaturating, while breathing spontaneously. After intubation, the endotracheal tube was wired to the front tooth. The patient was stable throughout the 3 -h operation and was 


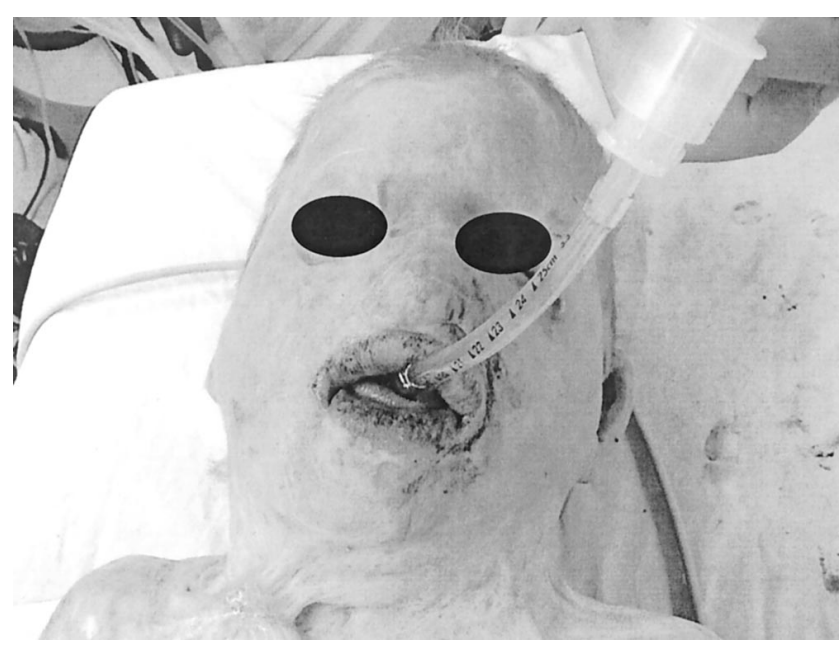

Figure 1. The endotracheal tube was wired to the front tooth of an 8 -yr-old boy with burn scar contractures of the nasal, facial, and neck region.

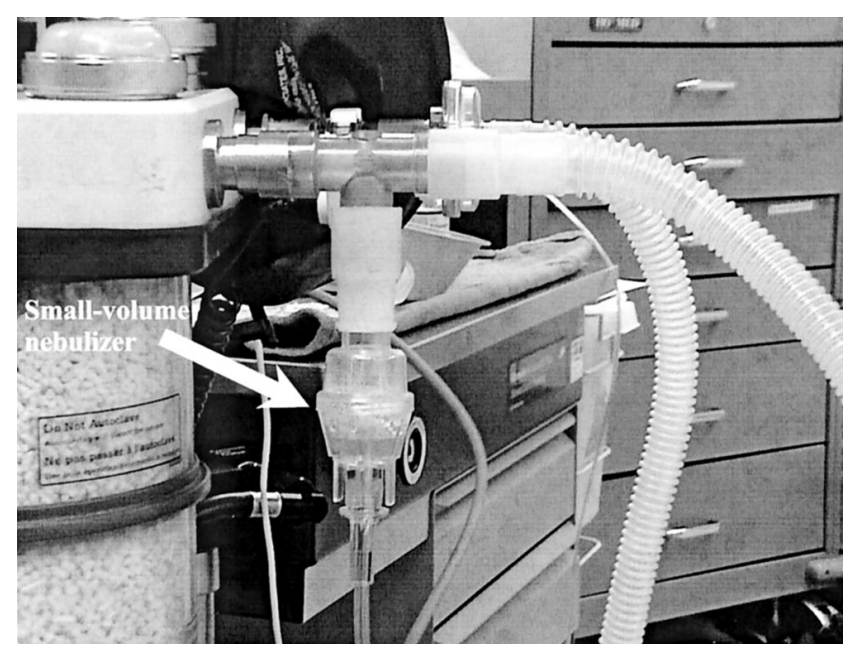

Figure 2. In-circuit nebulized lidocaine setup. A small-volume nebulizer was connected to the inspiratory limb of the circle system via a T-piece adapter.

tracheally extubated fully awake without difficulty at the end of the procedure.

One week later, the patient was rescheduled for another elective skin grafting. Anesthesia was successfully induced with $4 \%$ sevoflurane without apnea or desaturation. Again the patient received $5 \mathrm{~min}$ of nebulized $4 \%$ lidocaine with $4 \%$ end-tidal sevoflurane. Fiberoptic intubation was easily performed, and the child tolerated the procedure well, without coughing or desaturation. The patient was tracheally extubated at the end of surgery without complications and was discharged home 7 days later.

\section{Discussion}

This is the first described case of a difficult airway in a child with gross burn scarring in the facial and neck region in whom two successful fiberoptic intubations were performed after topicalization with in-circuit nebulized lidocaine. Upper airway manipulations, such as awake fiberoptic intubation, are often poorly tolerated in pediatric patients. Local and regional anesthesia techniques are frequently used to reduce the irritation associated with these procedures. These techniques are readily performed in awake or sedated adults but may require general anesthesia in children. It is prudent to maintain spontaneous respiration in an anesthetized child with a compromised airway. Inhaled induction in a child with a difficult airway is challenging, and the risks of airway obstruction, laryngospasm, and airway compromise are ever present, especially with premature intervention. Deep anesthesia under these circumstances may cause apnea and cardiovascular depression. Although the topical application of local anesthetics by using nebulization is one of the least invasive methods of anesthetizing the airway in awake adults, this approach may be difficult to effectively administer to uncooperative children.

In this case, the child had chromosome 9p duplication syndrome, which is characterized by mental retardation and craniofacial abnormalities. In addition, the patient had a limited range of mouth opening and neck motion because of scarring from a previous burn. Securing the airway in an uncooperative pediatric patient with severe facial and neck burns is a challenge, even to the most experienced anesthesiologist. The chosen anesthetic technique was influenced by previous failed attempts to secure a patent airway in the patient by direct laryngoscopy. Although the patient's airway was previously obtained with fiberoptic endoscopy via an LMA, in our opinion the potential for a life-threatening situation might be minimized in this particular patient by fiberoptic intubation under spontaneous respiration.

Nebulizing airways with local anesthetics through a small-volume nebulizer is routinely used in adults for airway management. However, we anticipated that this method would be time consuming and poorly tolerated in this unsedated patient. We hypothesized that simultaneously administering a volatile anesthetic with nebulized lidocaine would more effectively deliver lidocaine and provide better topical anesthesia for an uncooperative patient. With this technique, the patient was able to tolerate fiberoptic intubation while spontaneously breathing. IV lidocaine after sevoflurane induction has been shown to decease coughing and attenuate blood pressure increases after tracheal intubation in children $(1,2)$. Other combinations, such as propofol and sevoflurane, have been reported to decrease minimum alveolar anesthetic concentration requirements for intubation, but they may increase the risk of apnea (3). Convulsions from local anesthetic toxicity have been reported from the nebulization of lidocaine $(4,5)$. 
We recently conducted an in vitro study to evaluate the rate at which lidocaine is nebulized from smallvolume nebulizers at different oxygen flow rates (6). Approximately $0.2 \mathrm{~mL} / \mathrm{min}$ of $4 \%$ lidocaine is nebulized when the dispersing oxygen flow rate is 4 $\mathrm{L} / \mathrm{min}$. This $4 \mathrm{~L} / \mathrm{min}$ flow from the auxiliary oxygen, combined with a $4 \mathrm{~L} / \mathrm{min}$ flow of oxygen and $8 \%$ sevoflurane from the anesthesia machine, results in an $8 \mathrm{~L} / \mathrm{min}$ total gas flow with a $4 \%$ concentration of sevoflurane. During a 5-minute exposure to lidocaine nebulized in this fashion, the child would have received a maximum of $40 \mathrm{mg}$ of lidocaine $(1 \mathrm{~mL}$ of $4 \%$ lidocaine), which is much less than the maximum recommended dose of IV or nebulized lidocaine for any child weighing more than $10 \mathrm{~kg}$ (i.e., $<4 \mathrm{mg} / \mathrm{kg}$ lidocaine) (7).

Although this technique may facilitate fiberopticassisted intubation in anesthetized, spontaneously breathing children with compromised airways, further studies are warranted to determine the feasibility and efficacy of using an in-circuit nebulization system to deliver specific medications.

\section{References}

1. Jakobsen CJ, Ahlburg P, Holdgard HO, et al. Comparison of intravenous and topical lidocaine as a suppressant of coughing after bronchoscopy during general anesthesia. Acta Anaesthesiol Scand 1991;35:238-41.

2. Sayyid SS, Zalaket MI, Baraka AS. Intravenous lidocaine as adjuvant to sevoflurane anesthesia for endotracheal intubation in children. Anesth Analg 2003;96:1325-7.

3. Satsumae T, Watanabe S, Yamaguchi H. The effect of propofol infusion on minimum alveolar concentration of sevoflurane for smooth tracheal intubation. J Anesth 2002;16:28-33.

4. Groeben H, Schlicht M, Stieglitz S, et al. Both local anesthetics and salbutamol pretreatment affect reflex bronchoconstriction in volunteers with asthma undergoing awake fiberoptic intubation. Anesthesiology 2002;97:1445-50.

5. Efthimiou J, Higgenbottam T, Holt T, et al. Plasma concentrations of lignocaine during fibreoptic bronchoscopy. Thorax 1982;27: 68-71.

6. Tsui $\mathrm{BCH}$, Malherbe S. Nebulization of lidocaine with varied oxygen flow rates. Anesth Analg 2003;97:302.

7. Gjonaj ST, Lowenthal DB, Dozor AJ. Nebulized lidocaine administered to infants and children undergoing flexible bronchoscopy. Chest 1997;112:1165-9. 